\title{
Residual allergenicity of amino acid-based and extensively hydrolysed cow's milk formulas
}

\author{
M E Levin, ${ }^{1} \mathrm{MB}$ ChB, FCPaed (SA), MMed (Paed), Diploma Allergy (SA), PhD; D M Blackhurst ${ }^{2} \mathrm{PhD} ;$ F Kirstein, ${ }^{3}$ PhD; D Kok, ${ }^{4} \mathrm{BSc} ;$ \\ G F van der Watt, ${ }^{2}$ BSc, MB ChB, Dip Anaesth, MMed (Chem Path), FC Path (SA) Chem; A D Marais, ${ }^{2,5}$ MB ChB, FCP (SA) \\ ${ }^{1}$ Division of Allergology, Department of Paediatrics, Faculty of Health Sciences, University of Cape Town, South Africa \\ ${ }^{2}$ Division of Chemical Pathology, Department of Pathology, Faculty of Health Sciences, University of Cape Town, South Africa \\ ${ }^{3}$ Institute of Infectious Disease and Molecular Medicine, Division of Immunology, Faculty of Health Sciences, University of Cape Town; \\ and International Centre for Biotechnology and Genetic Engineering, Cape Town Component, South Africa \\ ${ }^{4}$ Division of Organic Chemistry, Faculty of Applied Sciences, Cape Peninsula University of Technology, Cape Town, South Africa \\ ${ }^{5}$ National Health Laboratory Service, Cape Town, South Africa
}

Corresponding author: M E Levin (michael.levin@uct.ac.za)

Background. Criteria for labelling infant feeds as suitable for the dietary management of cow's milk protein allergy (CMPA) rely on proving the hypoallergenicity of such feeds or clinical studies showing that the feeds are tolerated by $90 \%$ of children with proven CMPA. South African (SA) labelling legislation does not indicate what testing is necessary to prove hypoallergenicity.

Objectives. To evaluate all extensively hydrolysed cow's milk formulas and amino acid-based formulas available in SA for residual allergen content, protein size and amino-acid content.

Results. All amino-acid and extensively hydrolysed formulas were found to be similar in composition, with no residual cow's milk allergens detectable by enzyme-linked immunosorbent assay. Furthermore, proteins were absent and only small molecules in the size range of amino acids and possibly of very small oligopeptides were detected.

Conclusions. These findings indicate that the formulas are extremely likely to be compliant with the definition of hypoallergenicity as tolerance in $90 \%$ of proven sufferers from cow's milk allergy. The formulas may therefore be labelled as suitable for the dietary management of infants with CMPA.

S Afr Med J 2017;107(9):763-767. DOI:10.7196/SAMJ.2017.v107i9.12137

Breastmilk, the white liquid produced by the mammary glands, is the primary source of nutrition for infants before they are able to digest other types of food. Modified cow's milk formulas are the commonest breastmilk replacement formula, but are not always tolerated by infants with lactose intolerance or cow's milk protein allergy (CMPA). CMPA is an immune-mediated hypersensitivity reaction to cow's milk protein, ${ }^{[1]}$ which may be IgE mediated (presenting with urticaria, angio-oedema, and respiratory, abdominal and cardiac signs, and ranging from mild to anaphylactic reactions), or nonIgE mediated (presenting with delayed-type reactions, mainly in the gastrointestinal tract). The prevalence of CMPA is estimated at $2-4 \%$ worldwide, and preliminary data from the South African Food sensitisation and Food Allergy (SAFFA) study show a $6.4 \%$ prevalence of sensitisation and a $0.2 \%$ prevalence of challenge-proven CMPA in 1 - 3-year-old unselected urban infants. ${ }^{[2]}$

Cow's milk comprises casein and whey allergenic proteins. The casein allergens (collectively known as Bos d 8) comprise four different proteins (alpha-S1, alpha-S2, beta and kappa casein). The most important whey allergens include beta-lactoglobulin (Bos d 5 , the most abundant cow's milk whey protein, which occurs in the milk of many other species but is not present in human milk) and alpha-lactalbumin (Bos d 4). Bovine serum albumin (Bos d 6) and bovine immunoglobins (Bos $\mathrm{d} 7$ ) are less common allergens. The most common cow's milk antigens are intact proteins of a molecular weight between 14 and $150 \mathrm{kDa} \cdot{ }^{[3]}$ Cow's milk casein has a molecular weight of 19 - $25.2 \mathrm{kDa}$. Beta-lactoglobulin has a molecular weight of $18.3 \mathrm{kDa}$, a-lactalbumin $14.2 \mathrm{kDa}$, bovine serum albumin $66.3 \mathrm{kDa}$ and bovine immunoglobin $\mathrm{G} 150 \mathrm{kDa}$.
Cow's milk formulas are categorised according to the degree of hydrolysis into intact milk protein formulas, partially hydrolysed formulas (PHFs) or extensively hydrolysed formulas (EHFs). In addition, amino acid-based formulas (AAFs) manufactured from hydrolysis of nonmilk proteins or by combining individual amino acids are available. The nature of the formula may add additional indications to its use apart from its nutritional role, such as for dietary management as a replacement formula in children with established CMPA.

The term 'hypoallergenic formulas' was first used by Kleinman et al. ${ }^{[4]}$ to refer to formulas that are tolerated by $90 \%$ of subjects with proven cow's milk allergy with a 95\% confidence interval, and are therefore used for the treatment of cow's milk allergy. This definition was subsequently adopted by the World Allergy Organization (WAO) ${ }^{[5]}$

A statement of the European Society for Pediatric Allergology and Clinical Immunology ${ }^{[6]}$ stated in 1998 that infant formulas with a content of immunoreactive protein of $<1 \%$ of total nitrogen (which translates into the majority of peptides $<1.5 \mathrm{kDa}^{[7]}$ ) may be labelled as having 'reduced allergenicity'. In 2000, the American Academy of Pediatrics defined an extensively hydrolysed formula as a formula containing only peptides that have a molecular weight of $<3 \mathrm{kDa} .^{[8]}$ The guideline states that formulas with 'most of the nitrogen in the form of free amino acids and peptides $<1.5 \mathrm{kD}$ have been subjected to extensive clinical testing and meet the standard for hypoallergenicity'. In 2008, the American Academy of Pediatrics ${ }^{[9]}$ defined partially hydrolysed formulas as those that contain oligopeptides with a molecular weight generally $<5 \mathrm{kDa}$, extensively hydrolysed formulas as containing only peptides that have a molecular weight $<3 \mathrm{kDa}$ and 
free amino acid-based formulas as peptide-free mixtures of essential and non-essential amino acids. It is possible that individual amino acids can polymerise and form simple (usually di- or tri-) peptides.

Cross-linking of Fce receptor-bound IgE requires that an allergen contains at least two IgE-binding epitopes with a size of at least 15 amino acids. This implies that proteins with a size of $<3 \mathrm{kDa}$ are incapable of causing an allergic reaction. ${ }^{[10]}$ PHFs are not considered to be hypoallergenic according to Kleinman et al.' $\mathrm{s}^{[4]}$ definition; however, many EHFs have been proven to be hypoallergenic and all AAFs are hypoallergenic.

Current milk labelling practices are, however, confusing and contested. For example, many partially hydrolysed feeds are labelled as 'HA' and are referred to widely as 'hypoallergenic' because there has been some modification of their milk protein content by partial hydrolysis. Such partially hydrolysed feeds may have some efficacy in prevention of CMPA in high-risk subjects whose mothers are unable to breastfeed, but they are not suitable for subjects with confirmed CMPA, as they do not conform to the WAO definition of hypoallergenicity.

There have recently been changes to infant formula-labelling legislation in South Africa (SA). South African Department of Health Government Notice 35941 on 6 December 2012 (no. R991) ${ }^{[11]}$ legislated that mandatory statements on the labels of infant formula for special dietary management of infants with specific medical conditions replace claims of formula type or allergenicity (e.g. extensively or partially hydrolysed, allergen free, amino acid or hypoallergenic). Statements allowed include 'for the dietary management of infants with cow's milk allergy' or 'for the dietary management of infants with cow's milk allergy, multiple food protein allergies. The criteria by which such labelling is applied are not clear.

South African Department of Health Government Notice 32975 on 1 March 2010 (no. R146) ${ }^{[12]}$ is concerned with hypoallergenic, nonallergenic or allergen-free claims. The notice states that no claim shall be made that a foodstuff is hypoallergenic, non-allergenic or free of a common or uncommon allergen unless it is modified so as to reduce the endogenous antigens in such a way that it is not possible to detect the presence of any possible allergen with testing suitable for the specific allergen, or it has been tested to confirm the absence of such antigens using suitable testing.

The definition of what testing is suitable, which antigens should be tested for and in what form (e.g. size, degree of hydrolysis) is not covered by the legislation. With regard to infant formulas, candidates for testing include the native allergens themselves as well as the products of hydrolysis.

\section{Objectives}

To evaluate the residual allergen, protein, peptide and amino-acid content of AAFs and EHFs available in SA. This included:

- Testing for milk protein allergens (whole milk, $\beta$-lactoglobulin, casein, $\alpha$-lactalbumin) in AAFs and EHFs and comparing these with whole cow's milk and human breastmilk

- Testing for egg albumin, peanut and soy allergens in AAFs, EHFs, whole cow's milk and human breastmilk

- Testing for protein (peptides and amino acids) and the range in protein sizes in AAFs and EHFs and comparing these with whole cow's milk and human breastmilk.

\section{Methods}

Institutional review and ethical approval were sought and obtained (Human Research Ethics Committee, University of Cape Town, ref. no. 270/2014). Manufacturers of all companies marketing AAFs or EHFs in SA were notified of the research and permission was requested to identify their products by name. Where companies did not give permission for their products to be identified by name, they were referred to as the type of formula followed by a numeral if more than one of the same type of formula remained un-named (Table 1).

Where available, feeds were purchased from local pharmacies. Aminomed and Comidagen Plus (Nutr-e-Volution) were obtained directly from the manufacturer as they are sold via web-based ordering, and Neomino (Cipla) was obtained directly from the manufacturer as the feed had not yet been commercially launched.

The formulas were reconstituted according to the manufacturer's specifications under sterile conditions with sterile water ensuring no contamination, then aliquoted and frozen at $-20^{\circ} \mathrm{C}$ until thawed for specific analysis. Chemicals were of reagent grade. Deionised water was used for solutions. The samples were weighed on the same electronic balance and analyses were carried out in solutions according to the manufacturer's instructions, but were scaled down to practical volumes for use in the laboratory.

\section{Allergen content}

Formulas were tested in duplicate by enzyme-linked immunosorbent assay (ELISA) for whole-milk protein $(\alpha-, \beta$ - and $\kappa$-caseins, $\beta$-lactoglobulin), and individual $\beta$-lactoglobulin, casein, egg albumin, peanut and soy proteins using Ridascreen Fast test kits (R-Biopharm AG, Germany) and for a-lactalbumin (Bethyl Laboratories, USA).

These ELISA test kits were used for the quantitative analysis of residual milk allergen content in AAFs and EHFs, and tests were performed according to the manufacturer's protocol. Whole cow's milk and human breastmilk served as positive and negative controls, respectively. In addition, all samples were tested for the presence of soy, peanut and egg allergens.

\section{Protein concentration and sizes}

Conventional spectrophotometric protein assays are unreliable for small proteins and peptides, and two approaches were therefore used to describe these molecules by size.

- Coomassie Blue staining of proteins separated by sodium dodecyl sulfate polyacrylamide gel electrophoresis (SDS-PAGE). Each sample was reduced and denatured and underwent separation of amino acids, peptides and proteins in a $5-20 \%$ gradient polyacrylamide gel. ${ }^{[13]}$ Polyacrylamide gel was prepared in the laboratory in a Mini Protean II apparatus (Biorad, USA) according to Laemmli. ${ }^{[14]}$ Before application, $3 \mu \mathrm{L}$ of the sample were heated with $1 \%$ SDS and mercaptoethanol. Bromphenol blue was used with sucrose to load the sample. After running at $130 \mathrm{~V}$ until the blue dye approached the end of the gel, the gel was stained in Coomassie Blue solution and destained in methanol-acetic acid.

- Size-exclusion chromatography for amino acids and peptides. The reconstituted formula was filtered ( 0.45 microns) and analysed isocratically on an Agilent 1260 Infinity high-performance liquid chromatography (HPLC) system (Agilent, USA) that detected the absorption at selected wavelengths by diode array. The elution profiles were analysed for area under the curve (AUC) using the Masshunter software acquired with the machine. An Agilent SEC3 size exclusion column (Agilent, USA) was used with a flow rate of $0.35 \mathrm{~mL} / \mathrm{min}$ using phosphate-buffered saline. This separates molecules between $100 \mathrm{kDa}$ and $0.1 \mathrm{kDa}$. Calibration was carried out with selected amino acids and proteins. Adequate post-time was used to ensure no carry-over. HPLC employs a flow of molecules in solution through porous material that retards the progress of smaller molecules more than large molecules because the former venture into smaller spaces, increasing their 


\begin{tabular}{lll} 
Table 1. AAFs and EHFs tested & & \\
\hline Product & Intended age & Description \\
\hline AAFs & & \\
Aminomed (Nutr-e-Volution) & $<1$ year & Free amino-acid formula \\
Comidagen Plus (Nutr-e-Volution) & $>1$ year & Free amino-acid formula \\
Neocate LCP (Nutricia) & $<1$ year & Free amino-acid formula \\
Neocate Advance (Nutricia) & $>1$ year & Free amino-acid formula \\
Neomino (Cipla) & $<1$ year & Free amino-acid formula \\
EHFs & & \\
Alfare (Nestlé) & $<1$ year & Hydrolysed whey \\
Pepticate (Nutricia) & $<1$ year & Hydrolysed whey \\
Casein EHF 1 & $<1$ year & Hydrolysed casein \\
Similac Alimentum (Abbott) & $<1$ year & Hydrolysed casein \\
AAFs = amino acid-based formulas; EHFs = extensively hydrolysed formulas. & & \\
& &
\end{tabular}

travel distance relative to the large molecules that flow through the column with practically no delay. The molecular size markers were gamma-globulin $(150 \mathrm{kDa})$ at the void volume, albumin $(66 \mathrm{kDa})$, oxytocin $(1.00 \mathrm{kDa})$, tryptophan $(0.204 \mathrm{kDa})$ and glycine $(0.075 \mathrm{kDa})$.

\section{Results \\ Allergen content}

All AAF and EHF samples were negative for all milk allergens tested, with values below the detection limit of the assays (milk $2.5 \mathrm{mg} / \mathrm{kg}$, $\alpha$-lactalbumin $0.78 \mathrm{mg} / \mathrm{kg}, \beta$-lactoglobulin and casein $0.5 \mathrm{mg} / \mathrm{kg}$ ). The cow's milk sample, on the other hand, was positive for all milk allergens with values above the upper detection range of the assay (milk $67.5 \mathrm{mg} / \mathrm{kg}$, a-lactalbumin $50 \mathrm{mg} / \mathrm{kg}, \beta$-lactoglobulin and casein $13.5 \mathrm{mg} / \mathrm{kg}$ ), confirming correct performance of the tests. As expected, no cow's milk allergens were detected in human breastmilk. Furthermore, all samples tested negative for soy, peanut and egg allergens below the detection limit of the assays (peanut and soy $2.5 \mathrm{mg} / \mathrm{kg}$, egg albumin $0.5 \mathrm{mg} / \mathrm{kg}$ ).

\section{Protein electrophoresis}

After electrophoresis, staining with Coomassie Blue did not reveal any protein in the lanes containing reconstituted formulas. Proteins stained readily in cow's milk (lanes 10 and 12) as well as human milk (lane 11) (Fig. 1). Coomassie Blue stains proteins unreliably below 3 $\mathrm{kDA}$ and has a limit of detection at $0.1 \mu \mathrm{g}$ or even less. No protein was visible in any of the formula samples tested above the limit of detection of Coomassie Blue. The protein profiles differed between the cow's and human milk.

An attempt was made to detect small peptides and amino acids in a similar gel that was modified to $15 \%$ acrylamide to favour separation of smaller peptides, by derivatisation of carboxylic acid moieties with the fluorescent dye PDAM (1-pyrenyldiazomethane). This readily demonstrated glycine and tryptophan and conjugated material of identical size in the formulas, but larger peptides were not seen. In contrast, the cow's milk and breastmilk lanes lacked fluorescence in the range of single amino acids and small peptides, but there was faint fluorescence from the smallest size of protein seen in the Coomassiestained gels as well as in larger proteins (data not shown).

\section{HPLC with size exclusion}

HPLC with diode array detection of eluting compounds from a size exclusion column was used to discriminate molecules from amino acids through oligopeptides to proteins.

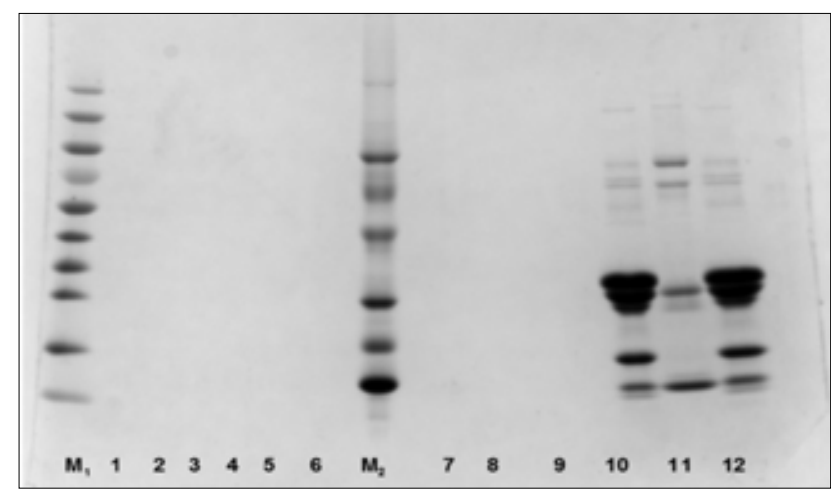

Fig. 1. Milk formula electrophoresis. (Lanes: 1 Alfare, 2 Allernova, 3 Pepticate, 4 Similac Alimentum, 5 Aminomed, 6 Comidagen Plus, 7 Neocate Advance, 8 Neocate LCP, 9 Neomino, 10 full-cream cow's milk, 11 breastmilk, 12 fat-free milk. $M_{1}$ molecular mass markers (small to large): 10, 15, $25,35,40,55,70,100,130,170 \mathrm{kDa} ; \mathrm{M}_{2}$ molecular mass markers (small to large): 14.3, 21.5, 30, 46, 69, 97.4, $200 \mathrm{kDa}$.)

The area of interest was determined by extrapolation from the markers: 13 minutes representing $3.02 \mathrm{kDa}, 14$ minutes $1.55 \mathrm{kDa}$ and 16 minutes $0.40 \mathrm{kDa}$. The peaks for the largest amino acid (tryptophan, $0.204 \mathrm{kDa}$ ) and the smallest (glycine, $0.075 \mathrm{kDa}$ ) were at 16.3 and 16.9 minutes, respectively.

The HPLC analysis covers the range of peptides below a molecular size of $3 \mathrm{kDa}$ that could be missed by the SDS-PAGE analysis. The HPLC analysis of very small molecules for sizes up to $\sim 0.4 \mathrm{kDa}$ could include the largest polymer of pentameric glycine and the simplest dimer could be one with tryptophan. Very small (oligo-) peptides can be considered to be in the size range of $0.4-1.5 \mathrm{kDa}$. The polymer with the largest number of amino acids in this range could be the triscidecamer of glycine and the lowest number of amino acids could be the pentamer of tryptophan. The larger peptides from 1.5 to $3 \mathrm{kDa}$ could be a 40 -mer of glycine to a 15 -mer of tryptophan. The detection of molecules by ultraviolet absorption favours amino acids, but may include other compounds.

The elution profiles of the formula samples contain material absorbing at molecular mass equivalents $>3 \mathrm{kDa}$. The SDS-PAGE analysis indicates that these absorbing substances are not proteins.

The overlay of all the elution profiles (Fig. 2) illustrates that the region between $3 \mathrm{kDa}$ and $0.4 \mathrm{kDa}$ is relatively clear of molecules absorbing at $214 \mathrm{~nm}$, with very similar patterns in the single aminoacid region between 16 and 18 minutes, indicated by tryptophan 
and glycine on the control elutions. The cow's milk and breastmilk samples contained no material at these low-molecular-weight ranges. This indicates that molecules larger than $3 \mathrm{kDa}$ were mostly absent in all formula samples tested.

The elution profiles were analysed for AUC in various segments representing $3-1.5 \mathrm{kDa}$ and $1.5-0.4 \mathrm{kDa}$, and the ratios were compared for these segments and the whole AUC for the region of $<3 \mathrm{kDa}$.

Although little difference was apparent in the molecular mass range between amino acids and $3 \mathrm{kDa}$, the AUC was analysed to crudely examine for differences between the two formula groups. The different formulations varied approximately four-fold in the 3.0 $1.5 \mathrm{kDa}$ peptide range, as well as in the $1.5-0.4 \mathrm{kDa}$ peptide range, whereas the variation was seven-fold in the $<0.4 \mathrm{kDa}$ (amino-acid) range. Comparison of the EHF group with the AAF group indicated

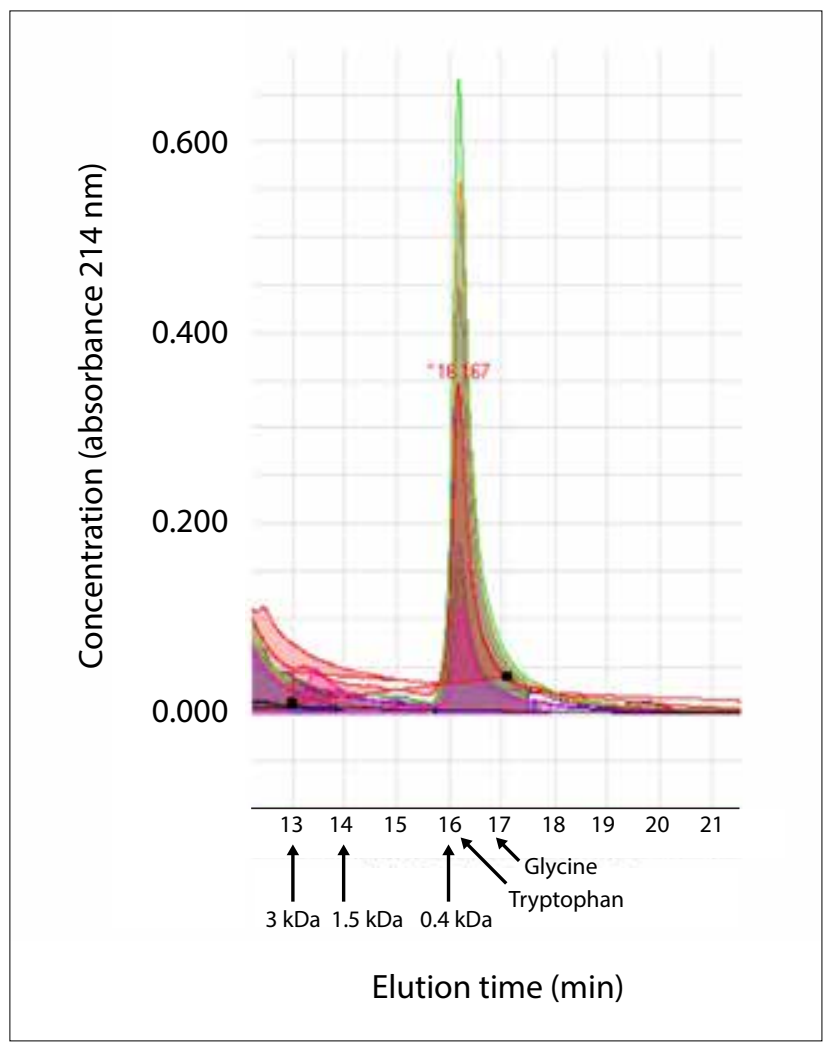

Fig. 2. Milk high-performance liquid chromatography peptide profile. $\left({ }^{\star}\right.$ Time after injection at which the peak was noted.) that the EHF preparations had a trend towards a greater AUC for the $0.4-1.5 \mathrm{kDa}$ size range than the AAF group (mean (standard deviation (SD)) 1383 (578) v. 687 (346); $p=0.064$ ). In the $<0.4 \mathrm{kDa}$ amino-acid region, the AAF group had a trend towards a higher AUC than the EHF group (mean 8453 (3 226) v. 4326 (2 442); $p=0.064$ ).

The proportion of amino acids $<0.4 \mathrm{kDa}$ in relation to the total range (peptides plus amino acids) was significantly higher in the AAF group than in the EHF group (mean (SD) 0.850 (0.062) v. 0.631 (0.126); $p=0.032)$. The EHF group also had a higher proportion of (presumed peptide) molecules $>0.4 \mathrm{kDa}$ compared with amino acids and small oligopeptides $(<0.4 \mathrm{kDa})$ than the AAF group (mean $0.630(0.295)$ v. $0.182(0.089)$, respectively; $p=0.032)$. Taken together, these findings suggest that EHFs may have more small oligopeptides than AAFs. According to these indirect criteria, $>50 \%$ of the EHF molecules were $<0.4 \mathrm{kDa}$, compared with $>75 \%$ of the AAF formulations.

Interestingly, there was no demonstrable material between 1.5 and $0.4 \mathrm{kDa}$ in cow's milk and human breastmilk, but the latter had demonstrable material between 0.4 and $3 \mathrm{kDa}$ (data not shown).

\section{Discussion}

It is extremely important that feeds for the dietary management of individuals with CMPA are free of residual allergenicity. Even trace amounts of native protein can cause significant reactions. ${ }^{[15]}$

The samples analysed in this study were randomly selected and are likely to be representative of the formulas in general. Since the formulas are manufactured according to set methods and with good quality control, it is unlikely that there will be significant differences from time to time.

All samples of AAFs and EHFs tested negative for all milk, soy, peanut and egg allergens in quantitative allergen-specific ELISA tests. This supports the conclusion that no protein antigens of a size large enough to bind to antibodies were present in the tested formulas.

Polyacrylamide gel analysis showed that none of the formulas tested contained detectable protein $>3 \mathrm{kDa}$, in contrast to the large amounts and variety of proteins in the cow's milk and breastmilk. The formulas lacked detectable large proteins and can therefore appropriately be termed protein-free, although technically small amounts of oligopeptides may be present.

The formulas vary significantly in the range of interest in molecular size, as described in Table 2. The detection of these molecules is not specific to amino acids, but was used to estimate differences in the low concentrations between amino acids and small oligopeptides. The AUC values for the molecular sizes between $3 \mathrm{kDa}$ and $0.4 \mathrm{kDa}$ are

Table 2. AUC for compounds $<3 \mathrm{kDa}$, and their ratios for selected regions detected by spectrophotometry

\begin{tabular}{lllllll}
\hline Feed type & Sample & $\begin{array}{l}\mathbf{3 - 1 . 5} \mathbf{~ k D a} \\
\text { (peptides) }\end{array}$ & $\begin{array}{l}\mathbf{1 . 5} \text { - } \mathbf{0 . 4} \mathbf{~ k D a} \\
\text { (peptides) }\end{array}$ & $\begin{array}{l}\mathbf{0 . 4} \mathbf{- 0 . 1} \text { kDa } \\
\text { (AAs) }\end{array}$ & $\begin{array}{l}\text { AAs divided by } \\
\text { (peptides + AAs) }\end{array}$ & Peptides/AAs \\
\hline EHF & Alfare & 1267 & 1969 & 5870 & 0.645 & 0.551 \\
EHF & EHF 1 & 503 & 1783 & 2694 & 0.541 & 0.849 \\
EHF & Pepticate & 789 & 813 & 1836 & 0.534 & 0.873 \\
EHF & Alimentum & 722 & 968 & 6904 & 0.803 & 0.245 \\
AAF & Aminomed & 285 & 1304 & 7785 & 0.830 & 0.204 \\
AAF & Comidagen Plus & 953 & 496 & 12664 & 0.897 & 0.114 \\
AAF & Neocate LCP & 338 & 514 & 8682 & 0.911 & 0.098 \\
AAF & Neocate Advance & 1048 & 563 & 9421 & 0.854 & 0.171 \\
AAF & Neomino & 638 & 558 & 3715 & 0.756 & 0.322 \\
& Mean & 727 & 996 & 6619 & 0.752 & 0.381 \\
& SD & 327 & 565 & 3489 & 0.145 & 0.304
\end{tabular}

$\mathrm{AUC}=$ area under the curve; $\mathrm{AAs}=$ amino acids; $\mathrm{EHF}=$ extensively hydrolysed formula; $\mathrm{AAF}=$ amino acid-based formula; $\mathrm{SD}=$ standard deviation. 
low relative to the amino-acid range in all samples. There is a trend towards more single amino-acid molecules in the AAFs than in the EHFs and for more oligopeptides in the EHFs than in the AAFs.

AAFs had a significantly higher proportion of amino acids in relation to the total range (peptides plus amino acids) than EHFs. Conversely, the EHFs had a higher proportion of oligopeptide molecules compared with amino acids than the AAFs. More than $50 \%$ of the $\mathrm{EH}$ molecules were $<0.4 \mathrm{kDa}$ compared with $>75 \%$ in the AAFs.

The clinical significance of the molecules between 0.4 and $3 \mathrm{kDa}$ is unknown. While it is possible that they may be immunogenic, they are subject to further digestion and modification in the body. It is theoretically possible to examine these molecules as antigens by performing reactions with plasma from patients suspected of allergy. It is unlikely that there is merit in analysing the species of oligomers of amino acids in the range of $3-0.1 \mathrm{kDa}$, unless a specific compound is thought to be particularly immunogenic. It is also possible that oxidatively modified amino acids could be present in the formulas. Such modification could happen during preparation or storage.

These data support the opinions expressed by the international Diagnosis and Rationale for Action against Cow's Milk Allergy ${ }^{[16]}$ food allergy guidelines and the recent South African consensus statement ${ }^{[1]}$ that extensively hydrolysed and amino acid formulas are suitable for the dietary management of cow's milk protein allergy. It is therefore appropriate that these formulas be labelled accordingly, in line with current legislation.

Unintended consumption of allergenic proteins can occur despite excellent labelling, for example due to an allergen's unlabelled inclusion in a food or cross-contamination during processing or after manufacture. ${ }^{[15]}$ Cross-contamination can be detected with ELISA testing to prove the presence of unintended allergens in both liquid and solid foodstuffs.

\section{Conclusions}

All AAFs and EHFs currently available in SA were found to be similar in composition, with an absence of proteins and a prominence of very small molecules. They have no residual allergenicity on ELISA testing for the native cow's milk protein and major allergenic components. Protein chains, where present, are of sufficiently small length to make it extremely likely that they will comply with the definition of hypoallergenicity as being tolerated by $90 \%$ of proven sufferers from cow's milk allergy, and they should therefore be labelled as suitable for the dietary management of subjects with cow's milk protein allergy.

Acknowledgements. None.

Author contributions. MEL conceived the project, sourced hydrolysed cow's milk formulas and amino-acid formulas, wrote up the article and reviewed the article; DMB performed chromatography and electrophoresis and contributed to writing and reviewing the article; FK designed the methods for allergen testing, performed allergen testing, wrote up sections of the article and reviewed the article; DK assisted with the performance of protein and amino-acid chromatography and its analysis and reviewed the article; GFvdW performed amino-acid and other analyses on the original formulations and reviewed the article; and ADM designed the methods for protein and amino-acid testing, performed protein and amino-acid testing, wrote up sections of the article and reviewed the article.

Funding. Funding for reagents and materials was obtained from Cipla Medpro.

Conflicts of interest. None.

1. Levin ME, Gray CL, Goddard E, et al., for the South African Food Allergy Working Group (SAFAWG). South African food allergy consensus document 2014. S Afr Med J 2015;105(1):62-65. https://doi org/10.7196/SAMJ.9098

2. Basera W, Botha M, Gray CL, et al. The South African Food sensitisation and Food Allergy (SAFFA) population-based study of IgE-mediated food allergy: Validity, safety and acceptability. Ann Allergy Asthma Immunol 2015;115(2):113-119. https://doi.org/10.1016/j.anai.2015.06.003

3. Steinman H, Ruden S. Native and recombinant allergen components. Uppsala, Sweden: X-O Graf Tryckeri AB, 2008.

4. Kleinman RE, Bahna S, Powell GF, Sampson HA. Use of infant formulas in infants with cow milk allergy: A review and recommendations. Pediatr Allergy Immunol 1991;2(4):986-997. https://doi. org/10.1111/j.1399-3038.1991.tb00200.x

5. Motala C, Fiocchi A. Cow's milk allergy in children. http://www.worldallergy.org/professional/ Motala C, Fiocchi A. Cow's milk allergy in children. http://www.worldallerg
allergic_diseases_center/cows_milk_allergy_in_children/ (accessed 29 July 2015).

6. Host A, Koletzko B, Dreborg S, et al. Dietary products used in infants for treatment and prevention of food allergy. Joint Statement of the European Society for Paediatric Allergology and Clinical Immunology (ESPACI) Committee on Hypoallergenic Formulas and the European Society for Paediatric Gastroenterology, Hepatology and Nutrition (ESPGHAN) Committee on Nutrition. Arch Dis Child 1999;81(1):80-84.

7. Meyer R, Venter C. Food hypersensitivity. In: Shaw V, Lawson M, eds. Clinical Paediatric Dietetics. 4th ed. Oxford: Wiley Blackwell, 2014:308-334.

8. American Academy of Pediatrics: Committee on Nutrition. Hypoallergenic infant formulae. Pediatrics 2000;106(2):346-349.

9. Greer FR, Sicherer SH, Burks AW, and the Committee on Nutrition and Section on Allergy and Greer FR, Sicherer SH, Burks AW, and the Committee on Nutrition and Section on Allergy and
Immunology. Effects of early nutritional interventions on the development of atopic disease in infants and children: The role of maternal dietary restriction, breastfeeding, timing of introduction of complementary foods and hydrolyzed formulas. Pediatrics 2008;121(1):183-192. https://doi. org/10.1542/peds.2007-3022

10. Huby RDJ, Dearman RJ, Kimber I. Why are some proteins allergens? Toxicol Sci 2000;55(2):235-246. https://doi.org/10.1093/toxsci/55.2.235

1. South Africa. Foodstuffs, Cosmetics and Disinfectants Act 54 of 1972. Regulations: Relating to foodstuffs for infants and young children (R991). Government Notice 35941, 2012.

12. South Africa. Foodstuffs, Cosmetics and Disinfectants Act 54 of 1972. Regulations: Relating to the . South Africa. Foodstuffs, Cosmetics and Disinfectants Act 54 of 1972. Regula
labelling and advertising of foodstuffs (R146). Government Notice 32975, 2010.

13. Swank RT, Munkres KD. Molecular weight analysis of oligopeptides by electrophoresis in polyacrylamide gel with sodium dodecyl sulfate. Anal Biochem 1971;39(2):462-477. https://doi. org/10.1016/0003-2697(71)90436-2

14. Laemmli UK. Cleavage of structural proteins during the assembly of the head of bacteriophage T4. Nature 1970;227(5259):680-685. https://doi.org/10.1038/227680a0

15. Levin ME, Motala C, Lopata AL. Anaphylaxis in a milk-allergic child following ingestion of soy formula cross-contaminated with cow's milk protein. Pediatrics 2005;116(5):1223-1225. https://doi org/10.1542/peds.2005-0020

16. Koletzko S, Niggemann B, Arato A, et al.; European Society of Pediatric Gastroenterology, Hepatology and Nutrition. Diagnostic approach and management of cow's-milk protein allergy in infants and and Nutrition. Diagnostic approach and management of cow's-milk protein allergy in infants and
children: ESPGHAN GI Committee practical guidelines. J Pediatr Gastroenterol Nutr 2012;55(2):221229. https://doi.org/10.1097/MPG.0b013e31825c9482

Accepted 18 April 2017. 